\title{
Biomechanical integrity score of the female pelvic floor
}

\author{
Vladimir Egorov ${ }^{1}(1) \cdot$ Heather van Raalte ${ }^{2} \cdot$ Peter Takacs $^{3} \cdot$ S. Abbas Shobeiri ${ }^{4} \cdot$ Vincent Lucente $^{5} \cdot$ Lennox Hoyte $^{6}$
}

Received: 24 November 2021 / Accepted: 3 February 2022 / Published online: 1 March 2022

(c) The Author(s) 2022

\begin{abstract}
Introduction and Hypothesis The aim of this study is to develop and validate a new integral parameter, the Biomechanical Integrity score (BI-score), for the characterization of the female pelvic floor.

Methods A total of 253 subjects with normal and pelvic organ prolapse (POP) conditions were included in the multi-site observational, case-control study; 125 subjects had normal pelvic floor conditions, and 128 subjects had POP stage II or higher. A Vaginal Tactile Imager (VTI) was used to acquire and automatically calculate 52 biomechanical parameters for eight VTI test procedures (probe insertion, elevation, rotation, Valsalva maneuver, voluntary muscle contractions in two planes, relaxation, and reflex contraction). Statistical methods were applied ( $t$-test, correlation) to identify the VTI parameters sensitive to the pelvic conditions.

Results Twenty-six parameters were identified as statistically sensitive to POP development. They were subdivided into five groups to characterize (1) tissue elasticity, (2) pelvic support, (3) pelvic muscle contraction, (4) involuntary muscle relaxation, and (5) pelvic muscle mobility. Every parameter was transformed to its standard deviation units against the patient age similar to T-score for bone density. Linear combinations with specified weights led to the composition of five component parameters for groups (1)-(5) and the BI-score in standard deviation units. The $p$-value for the BI-score has $p=4.3 \times 10^{-31}$ for POP versus normal conditions. A reference BI-score curve against age for normal pelvic floor conditions was defined. Conclusions Quantitative transformations of the pelvic tissues, support structures, and functions under diseased conditions may be studied with the BI-score in future research and practical applications.
\end{abstract}

Keywords Pelvic organ prolapse $\cdot$ Tissue elasticity $\cdot$ Pelvic support $\cdot$ Pelvic muscle strength $\cdot$ Muscle relaxation $\cdot$ Muscle mobility $\cdot$ Biomechanical integrity score

\section{Introduction}

Pelvic organ prolapse (POP) is a common condition in women and often associated with concomitant pelvic floor disorders, including urinary and fecal incontinence, pelvic pain, voiding, and sexual dysfunctions, which may adversely

Vladimir Egorov

egorov@ tactile-imaging.com

1 Advanced Tactile Imaging, 1457 Lower Ferry Rd, Trenton, NJ 08618, USA

2 Princeton Urogynecology, Princeton, NJ, USA

3 Eastern Virginia Medical School, Norfolk, VA, USA

4 INOVA Fairfax Hospital, Falls Church, VA, USA

5 The Institute for Female Pelvic Medicine \& Reconstructive Surgery, Allentown, PA, USA

6 The Pelvic Floor Institute, Tampa, FL, USA affect the quality of life [1]. The current clinical practice for the assessment of pelvic floor disorders is often limited to the evaluation of surface anatomy and manual palpation. The Pelvic Organ Prolapse Quantification (POP-Q) system is widely used for describing and staging pelvic support [2], the Pelvic Floor Distress Inventory (PFDI) and PFDI-20 are recommended by the International Consultation on Incontinence as grade A for assessing pelvic floor dysfunction [3], and the Female Sexual Function Index (FSFI) is used for diagnosing sexual dysfunction in women [4]. In severe or complicated cases, ultrasound, magnetic resonance imaging (MRI), and $\mathrm{X}$-ray imaging may be used for additional evaluation. Bladder and rectum function tests, such as urodynamics, manometry, or defecography, might also be employed [5-8].

The true etiology of POP and variations observed among individuals are not entirely understood. These disorders are thought to share common pathogeneses, tissue elasticity changes, weakening of the connective 
support tissues, and pelvic muscle dysfunction [9-14]. Logically, proposing a biomechanical assessment and characterization of the female pelvic floor could give rise to important information in clinical practice. However, ultrasound and MRI elastography, as well as functional imaging of the pelvic floor, did not obtain appropriate acceptance in urogynecology. There is a significant gap in the biomechanical and functional research of the female pelvic floor [15-20]. The PUBMED database search for "(biomechanical[Title/Abstract]) AND (functional[Title/ Abstract]) AND (pelvic floor[Title/Abstract])" results in a total of only 16 publications for all the time.

The Vaginal Tactile Imager (VTI) was developed to provide biomechanical mapping of the pelvic floor with a vaginal probe [21]. A set of new clinical markers/parameters has been proposed for the biomechanical characterization of the pelvic floor conditions [20, 22]. This set includes 52 parameters automatically calculated as a result of the completion of eight examination procedures (tests). However, this approach did not gain momentum among urogynecologists because of the long list of parameters and difficulties in their explanations to clinicians and patients. To make biomechanical mapping in urogynecology more accessible and useful, further work is required on the development of a shorter list of easily understandable and practical parameters.

The aim of this article is to report the development and validation of a new integral parameter, the Biomechanical Integrity score (BI-score), for the characterization of female pelvic floor conditions.

\section{ilmaging is a medical imaging modality that translates the sense of touch into a digital imageMaterials and Methods}

\section{Definitions}

Tactile Imaging is a medical imaging modality that translates the sense of touch into a digital image [21]. The tactile image is a function of $P(x, y, z)$, where $P$ is the pressure on the soft tissue surface under applied deformation and $x, y$, and $z$ are the coordinates where $P$ was measured. The tactile image is a pressure map on which the direction of tissue deformation must be specified.
Functional Tactile Imaging translates muscle activity into dynamic pressure pattern $P(x, y, t)$ for an area of interest, where $t$ is time and $x$ and $y$ are coordinates where the pressure $P$ was measured. It may include: (1) muscle voluntary contraction, (2) involuntary reflex contraction, (3) involuntary relaxation, and (4) specific maneuvers.

\section{Biomechanical Mapping = Tactile Imaging + Functional Tactile Imaging}

A tactile imaging probe has a pressure sensor array mounted on its face that acts similarly to human fingers during a clinical examination, deforming the soft tissue and detecting the resulting changes in the pressure pattern on the surface. The sensor head is moved against or over the surface of the tissue to be studied, and the pressure response is measured at multiple locations along the tissue. The results are used to generate images that show pressure distribution over the area of the tissue under study. The tactile image $P(x, y, z)$ reveals tissue or organ anatomy and elasticity distribution [23, 24].

\section{Vaginal Tactile Imager}

The Vaginal Tactile Imager (VTI), model 2S, was used for biomechanical mapping of the pelvic floor. The VTI probe, as shown in Fig. 1, is equipped with 96 pressure (tactile) sensors spaced consecutively on both sides of the probe, an orientation sensor, and temperature controllers to provide the probe temperature close to a human body before the examination. During the clinical procedure, the probe is used to acquire pressure responses from two opposite vaginal walls along the vagina. The VTI data are sampled from the probe sensors and presented on the VTI display in real time. The resulting pressure maps (tactile images) of the vagina integrate all the acquired pressure and positioning data for each of the pressure sensing element during vaginal wall deformation and pelvic muscle contraction. Lubricating jel is used for patient comfort. It is also utilized to provide reproducible boundary/contact conditions with deformed tissues.

The VTI examination procedure consists of eight tests (see Appendix 1). The spatial gradients $\partial P(x, y) / \partial y$ (changes of pressure along the tissue deformation per $1 \mathrm{~mm}$ ) for anterior and posterior compartments are calculated within the acquired tactile images in Test 1 and 2; the $y$-coordinate
Fig. 1 Vaginal probe. Pressure sensors are aligned on the outer surfaces of the probe (highlighted in the image)

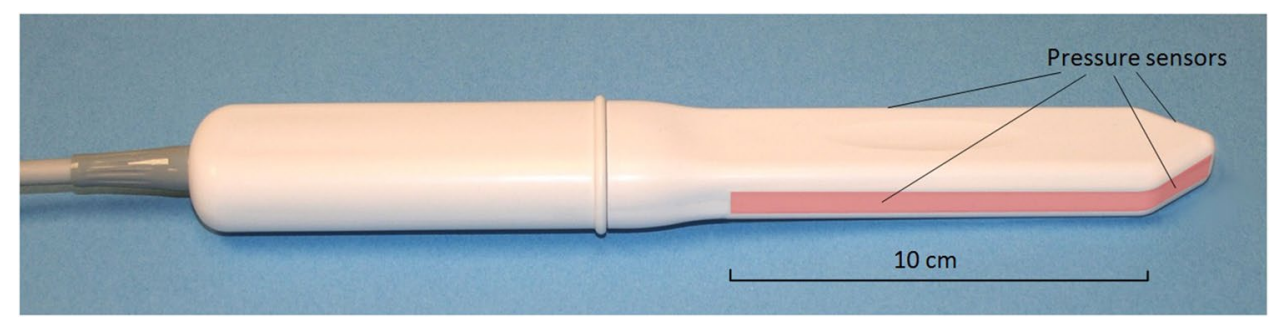


is directed orthogonally from the vaginal channel coming through the anterior-posterior compartments, and the $x$-coordinate is located on the vaginal channel.

The VTI probe is calibrated with reference pressure sensors (Honeywell) immediately before every subject's examination. The VTI absolute measurement accuracy is as follows: $\pm 0.2 \mathrm{kPa}$ within $0-10 \mathrm{kPa}$ range, $\pm 0.5 \mathrm{kPa}$ at $25 \mathrm{kPa}$, and $\pm 1.0 \mathrm{kPa}$ at $60 \mathrm{kPa}$. The VTI relative pressure measurement accuracy lies in the range of $\pm 0.05 \mathrm{kPa}$ to $\pm 0.1 \mathrm{kPa}$. The intra- and inter-observer reproducibility of the vaginal tactile imaging was reported earlier [25]. The VTI pressure measurement resolution is $0.001 \mathrm{kPa}$. The VTI absolute measurement accuracy for probe orientation is $\pm 0.5^{\circ}$ and $\pm 0.1{ }^{\circ} \mathrm{C}$ for measuring the temperature inside the probe on the surface of the pressure sensors. The tactile images and muscle contraction patterns are visualized with a resolution of $1 \mathrm{~mm}$ [21].

\section{Biomechanical parameters}

The full list of 52 VTI biomechanical parameters, their interpretation, and anatomical assignments of the targeting/ contributing pelvic structures into the specified parameters are presented in Appendix 2.

\section{Study population}

The analyzed dataset in this study includes subjects with normal pelvic floor conditions and POP stage II or higher from three VTI clinical studies with identical VTI examination procedures. These subjects were examined with the VTI in the scope multi-site observational, case-control studies completed from September 2014 to December 2018 (clinical trials identifiers NCT02294383 and NCT02925585) and study with VTI11 protocol (June 2020-September 2021). The recruitment sites were Princeton Urogynecology (Princeton, NJ), Eastern Virginia Medical School (Norfolk, VA), INOVA Fairfax Hospital (Falls Church, VA), The Institute for Female Pelvic Medicine and Reconstructive Surgery (Allentown, PA), and The Pelvic Floor Institute (Tampa, FL). It was important that all the analyzed subjects have not had any prior pelvic surgery. Table 1 presents the mean and standard deviation for the subject age, parity, weight, and height separately for normal and POP groups. All clinical protocols were approved by the Institutional Review Board (Western IRB and local IRB as required), and written informed consent was obtained from all the subjects enrolled in the studies. This clinical research was done in compliance with the Health Insurance Portability and Accountability Act. The VTI examination data for the eight tests (see Appendix 1) were obtained and recorded at the time of the scheduled urogynecologic visits.

The total study workflow comprised the following steps: (1) recruiting women who had not previously had a pelvic surgery and had normal pelvic floor conditions (no POP, stage 0) or had POP stage II or higher; (2) acquiring clinical diagnostic information related to the case by standard clinical means; (3) performing a VTI examination in lithotomic position; (4) analyzing VTI data. Study exclusion criteria were: (1) active skin infection or ulceration within the vagina; (2) presence of a vaginal septum; (3) active cancer of the colon, rectal wall, cervix, vaginal, uterus, or bladder; (4) ongoing radiation therapy for pelvic cancer; (5) impacted stool; (6) significant pre-existing pelvic pain, including levator ani syndrome, severe vaginismus, or vulvodynia; (7) severe hemorrhoids; (8) significant circulatory or cardiac conditions that could cause excessive risk from the examination as determined by the attending physician; (9) current pregnancy. In the VTI11 protocol, which targets the normal pelvic conditions, two exclusion criteria were added as follows: (1) the woman is a regular patient visiting the urogynecology clinic $(2+$ times during the last year); (2) the patient has cognitive impairment. Prior to the VTI examination, a standard physical examination was performed, including a bimanual pelvic examination and Pelvic Organ Prolapse Quantification (POP-Q) [2].

\section{Statistical methods}

A total of 52 biomechanical parameters were calculated automatically by the VTI software version 2018.54.4.0 per each of the 253 analyzed VTI examination data. The two-sample $t$-test $(p<0.05)$ was employed to test the null hypothesis that the data in normal and POP groups have equal means and equal variances. The alternative hypothesis
Table 1 Demographic data for the studied groups

\begin{tabular}{lllllll}
\hline & $\begin{array}{l}\text { Norm } \\
\text { mean } \\
(n=125)\end{array}$ & $\begin{array}{l}\text { Norm SD } \\
(n=125)\end{array}$ & POP mean $(n=128)$ & $\begin{array}{l}\text { POP SD } \\
(n=128)\end{array}$ & $\begin{array}{l}100 \% *(\text { POP- } \\
\text { Norm }) / \text { Norm }\end{array}$ & $\begin{array}{l}\text { Norm } \\
\text { vs. POP } \\
p \text {-value }\end{array}$ \\
\hline Patient age, years & 36.0 & 15.0 & 65.5 & 11.5 & $81.9 \%$ & $2.6 \mathrm{E}-35$ \\
Patient parity & 0.9 & 1.0 & 2.4 & 1.1 & $166.7 \%$ & $2.5 \mathrm{E}-25$ \\
Patient weight, kg & 69.5 & 14.3 & 70.0 & 12.9 & $0.7 \%$ & $7.6 \mathrm{E}-01$ \\
Patient height, cm & 163.2 & 9.6 & 162.3 & 7.7 & $-0.6 \%$ & $4.4 \mathrm{E}-01$ \\
Patient POP-Q & 0 & - & Stage II+ & - & - & - \\
\hline
\end{tabular}


is that the data in these groups come from populations with unequal means. $P$-values for testing hypothesis were calculated. Pearson's linear correlation coefficients $(r)$ were calculated among 52 VTI parameters, each parameter against all other 51 parameters.

For the visual evaluation of the analyzed data distributions, we used notched boxplots [26] showing a confidence interval for the median value (central vertical line), $25 \%$ and $75 \%$ quartiles. The spacing between the different parts of the box helps to compare variance. The boxplot also determines skewness (asymmetry) and outlier (cross). The statistical functions of MATLAB, version R2021a (MathWorks, MA), were used for the data analysis.

\section{Results}

Among the 253 subjects analyzed in this study, $125 \mathrm{had}$ normal pelvic floor conditions and 128 had POP stage II or higher. The pelvic floor conditions were categorized by the stage of the prolapse based on the maximum stage from anterior, posterior, and uterine prolapse. Employing this approach, we found that 68 subjects had POP stage II, 57 had stage III, and 3 had stage IV.

At first, we aimed at selecting VTI parameters with significant changes at POP versus the normal pelvic conditions. Two specific quantitative criteria were imposed on such selection: (1) a $t$-test $p<0.05$ for the sub-set data of 128 POP cases against the sub-set data for 125 normal cases and (2) a correlation coefficient $r<0.85$ with all other parameters. The first criteria passed 40 parameters, both first and second 26 parameters. Figure 2 presents the boxplots, and Table 2 shows the numerical data for these 26 selected VTI parameters responsive to POP and not highly correlated with each other. For consistency, the numbering of the VTI parameters in this article is kept exactly as in earlier publications [22].

The parameters listed in Table 2 have different units (see column 5 in Table 2). The next step was to bring all the selected parameters to uniform units to allow their arithmetic combination. Among various possible options, the preference to the units of standard deviation was provided (see the explanation pertaining to such selection in the Discussion section). All VTI data were transformed according to Eq. 1 below.

$P s d_{n}^{i}=\left(P o_{n}^{i}-P a_{n}\right) / S D_{n}$

Here, $P o_{n}^{i}$ is an original value of the $n$ parameter for $i$ subject; $P a_{n}$ is an arithmetic average of the $n$ parameter for subjects aged 18-39 years in the group with normal pelvic conditions (92 out of 125 subjects); $S D_{n}$ is a standard deviation for the $n$ parameter for 125 subjects in the group with normal pelvic conditions; $P s d_{n}^{i}$ is the transformed value of the $n$ parameter for $i$ subject in units of standard deviation.

Now, we can combine the parameters expressed in units of standard deviation using a linear operation of addition. First, the 26 selected parameters were subdivided into five groups. We may call them by the five components to characterize: (1) tissue elasticity, (2) pelvic support, (3) pelvic muscle contraction, (4) muscle relaxation, and (5) muscle mobility (see Fig. 3). Component 1 comprises eight parameters with equal weights of $0.125(8 \times 0.125=1.0)$, component 2 comprises five parameters with equal weights of 0.2 , component 3 consists of eight parameters with weights of 0.1 and 0.2 , component 4 comprises two parameters with weight of 0.5 , and component 5 consists of three parameters with weight of 0.2 and 0.4 . Basically, the same (equal) weights within the components were used to provide 1.0 at summation. In two components (muscle contraction and mobility), the preferences in weights were given to the VTI parameters with the highest $p$-values. Finally, these five components create the Biomechanical Integrity score (BI-score) with equal weights of 0.2 as shown in Fig. 3.

All the BI-score data for 253 subjects analyzed here can be visualized on one graph as a function of the subject's age (see left panel in Fig. 4). A second order polynomial fit for the BI-score values against the subject's age for all the 125 subjects in the group with normal pelvic conditions is presented by a blue line (reference line) in Fig. 4 . The grey dashed lines show \pm 1.0 standard deviation from the reference line. The right panel in Fig. 4 shows the same BI-score data in two boxplots for normal and POP pelvic conditions. One may observe significant separation between these two groups; the $t$-test gives $p=4.3 \times 10^{-31}$ for these two groups.

Figure 5 illustrates the idea that the BI-score and all its five components can be presented on one graph with the same vertical axis (standard deviation) because all of them have the same units. Three colored backgrounds denote the zones of certainly normal pelvic conditions (green), transition (blue), and POP (red).

\section{Discussion}

The BI-score has certain similarities with the T-score developed for bone density characterization. As bone mineral density (BMD) technology evolved in the twentieth century, it became clear that BMD expressed in raw units would be difficult to interpret. Ideally, for BMD measurements to be clinically useful, they should be presented in terms that are readily understandable by patients and clinicians as well as independent of the densitometer used or the skeletal site measured. The T-score was suggested to 

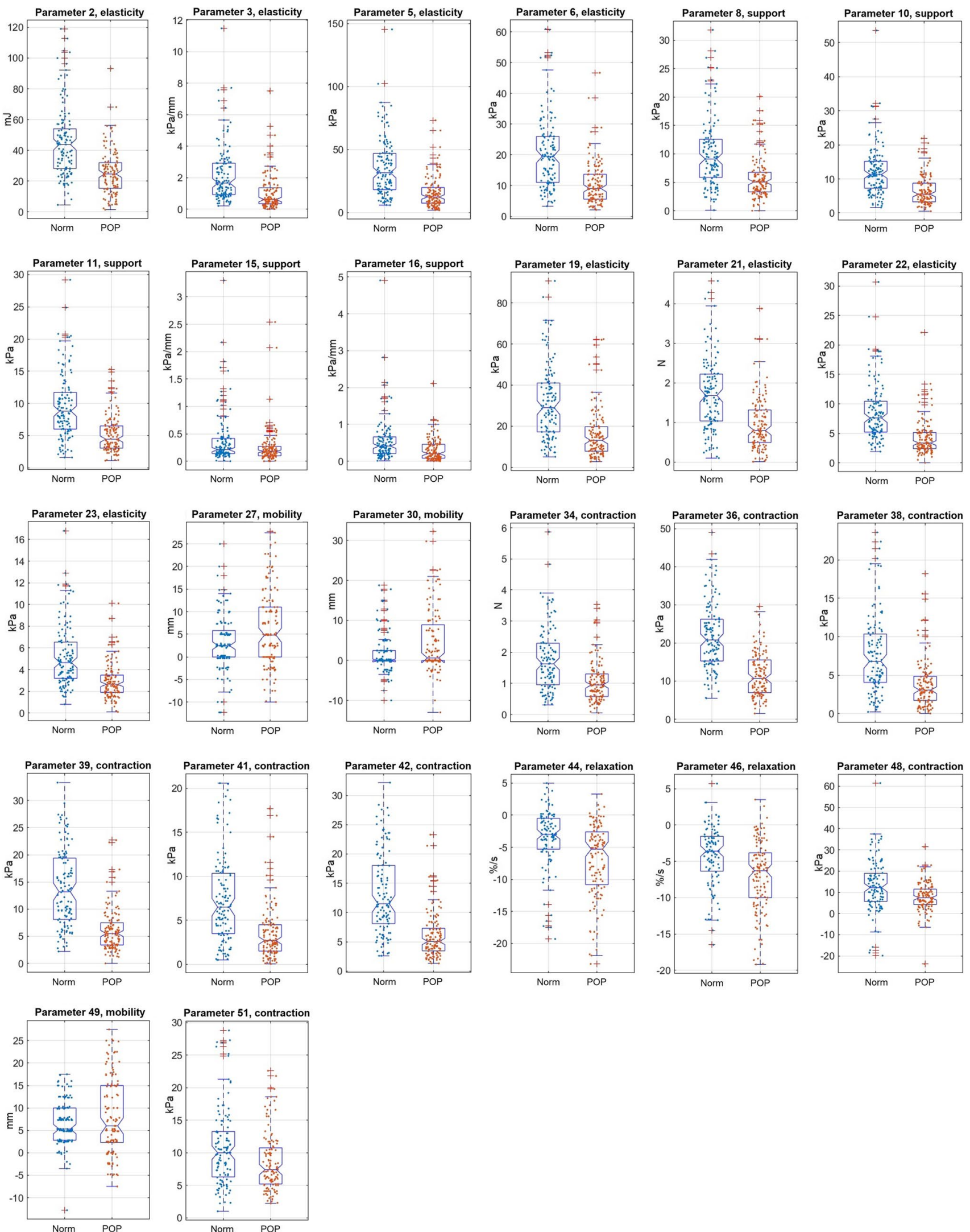

Fig. 2 Boxplots for 26 identified VTI parameters which demonstrate statistically significant sensitivity to POP conditions and not highly correlated with each other 
Table 2 VTI parameters and BI-score sensitivity to POP conditions

\begin{tabular}{|c|c|c|c|c|c|c|c|c|c|c|}
\hline $\begin{array}{l}\text { Param- } \\
\text { eter } \\
\text { no.* }\end{array}$ & Test no. & BI-score component & Parameter weight & Units & $\begin{array}{l}\text { Norm } \\
\text { mean } \\
(n=125)\end{array}$ & $\begin{array}{l}\text { Norm SD } \\
(n=125)\end{array}$ & $\begin{array}{l}\text { POP } \\
\text { mean } \\
(n=128)\end{array}$ & $\begin{array}{l}\text { POP SD } \\
(n=128)\end{array}$ & $\begin{array}{l}100 \% *(\mathrm{POP}- \\
\text { Norm)/Norm }\end{array}$ & $\begin{array}{l}\text { Norm } \\
\text { vs. POP } \\
p \text {-value }\end{array}$ \\
\hline 2 & 1 & Elasticity & 0.125 & $\mathrm{~mJ}$ & 45.4 & 22.4 & 25.4 & 14.7 & $-44.1 \%$ & $4.1 \mathrm{E}-15$ \\
\hline 3 & 1 & Elasticity & 0.125 & $\mathrm{kPa} / \mathrm{mm}$ & 2.18 & 1.76 & 1.05 & 1.14 & $-51.9 \%$ & 5.8E-09 \\
\hline 5 & 1 & Elasticity & 0.125 & $\mathrm{kPa}$ & 35.7 & 23.1 & 16.0 & 12.6 & $-55.1 \%$ & $3.6 \mathrm{E}-15$ \\
\hline 6 & 1 & Elasticity & 0.125 & $\mathrm{kPa}$ & 20.6 & 12.0 & 10.8 & 7.08 & $-47.5 \%$ & $1.2 \mathrm{E}-13$ \\
\hline 8 & 2 & Support & 0.200 & $\mathrm{kPa}$ & 10.1 & 6.09 & 5.77 & 3.79 & $-43.3 \%$ & $6.8 \mathrm{E}-11$ \\
\hline 10 & 2 & Support & 0.200 & $\mathrm{kPa}$ & 12.2 & 7.33 & 6.61 & 4.25 & $-46.0 \%$ & $1.1 \mathrm{E}-12$ \\
\hline 11 & 2 & Support & 0.200 & $\mathrm{kPa}$ & 9.52 & 5.07 & 5.22 & 2.87 & $-45.2 \%$ & 5.3E-15 \\
\hline 15 & 2 & Support & 0.200 & $\mathrm{kPa} / \mathrm{mm}$ & 0.38 & 0.47 & 0.25 & 0.31 & $-35.6 \%$ & $6.8 \mathrm{E}-03$ \\
\hline 16 & 2 & Support & 0.200 & $\mathrm{kPa} / \mathrm{mm}$ & 0.55 & 0.60 & 0.30 & 0.30 & $-45.9 \%$ & $3.6 \mathrm{E}-05$ \\
\hline 19 & 3 & Elasticity & 0.125 & $\mathrm{kPa}$ & 31.4 & 17.7 & 16.1 & 12.3 & $-48.7 \%$ & 5.7E-14 \\
\hline 21 & 3 & Elasticity & 0.125 & $\mathrm{~N}$ & 1.71 & 0.90 & 0.96 & 0.67 & $-43.6 \%$ & $1.3 \mathrm{E}-12$ \\
\hline 22 & 3 & Elasticity & 0.125 & $\mathrm{kPa}$ & 8.66 & 4.90 & 4.26 & 3.01 & $-50.8 \%$ & $8.3 \mathrm{E}-16$ \\
\hline 23 & 3 & Elasticity & 0.125 & $\mathrm{kPa}$ & 5.16 & 2.82 & 2.95 & 1.64 & $-42.9 \%$ & $4.1 \mathrm{E}-13$ \\
\hline 27 & 4 & Mobility & 0.400 & $\mathrm{~mm}$ & 3.26 & 6.17 & 5.83 & 8.54 & $79.0 \%$ & 7.9E-03 \\
\hline 30 & 4 & Mobility & 0.400 & $\mathrm{~mm}$ & 1.71 & 4.97 & 4.56 & 7.86 & $167.0 \%$ & 8.9E-04 \\
\hline 34 & 5 & Contraction & 0.200 & $\mathrm{~N}$ & 1.77 & 1.01 & 1.05 & 0.66 & $-40.3 \%$ & $1.9 \mathrm{E}-10$ \\
\hline 36 & 5 & Contraction & 0.200 & $\mathrm{kPa}$ & 21.8 & 8.55 & 11.6 & 5.76 & $-46.7 \%$ & $1.2 \mathrm{E}-23$ \\
\hline 38 & 6 & Contraction & 0.100 & $\mathrm{kPa}$ & 7.55 & 5.12 & 3.79 & 3.17 & $-49.8 \%$ & 2.3E-11 \\
\hline 39 & 6 & Contraction & 0.100 & $\mathrm{kPa}$ & 13.7 & 7.06 & 6.10 & 3.92 & $-55.7 \%$ & $4.9 \mathrm{E}-22$ \\
\hline 41 & 6 & Contraction & 0.100 & $\mathrm{kPa}$ & 7.29 & 4.92 & 3.58 & 3.11 & $-50.9 \%$ & $9.7 \mathrm{E}-12$ \\
\hline 42 & 6 & Contraction & 0.100 & $\mathrm{kPa}$ & 13.2 & 6.97 & 6.06 & 3.93 & $-54.4 \%$ & $2.0 \mathrm{E}-20$ \\
\hline 44 & 7 & Relaxation & 0.500 & $\% / \mathrm{s}$ & -3.68 & 4.75 & -6.99 & 5.70 & $90.0 \%$ & 3.0E-06 \\
\hline 46 & 7 & Relaxation & 0.500 & $\% / \mathrm{s}$ & -4.20 & 4.10 & -6.95 & 4.79 & $65.3 \%$ & $5.0 \mathrm{E}-06$ \\
\hline 48 & 8 & Contraction & 0.100 & $\mathrm{kPa}$ & 12.9 & 12.2 & 7.84 & 6.86 & $-39.4 \%$ & $8.5 \mathrm{E}-05$ \\
\hline 49 & 8 & Mobility & 0.200 & $\mathrm{~mm}$ & 6.55 & 4.95 & 8.01 & 8.55 & $22.4 \%$ & 4.8E-02 \\
\hline 51 & 8 & Contraction & 0.100 & $\mathrm{kPa}$ & 10.8 & 6.00 & 8.59 & 4.58 & $-20.5 \%$ & $1.4 \mathrm{E}-03$ \\
\hline
\end{tabular}

*Parameter numbering as in Appendix 2

simplify the interpretation of the bone density result and to avoid the use of raw BMD values. The T-score was defined as the difference between a BMD and the expected young normal value divided by the population standard deviation; it is similar to Eq. 1 in this article. In a rare moment of scientific convergence among instrument manufacturers, the T-score was quickly adopted as a consistent output parameter for all densitometry devices [27]. With the World Health Organization (WHO) Study Group report, the role of the T-score for osteoporosis diagnosis was solidified [28]. Currently, osteoporosis researchers and clinicians use the T-score as the most relevant diagnostic value from a bone density examination.

The BI-score is a composite score that consists of five components as shown in Fig. 3. These five components bring different aspects of biomechanical characterization of the pelvic floor. Due to the exclusion of the highly correlated original VTI parameters with $r \geq 0.85$, the mutual correlation coefficients have an average value of $r=0.27$, which is considered as low or negligible correlation. It is important to note that the tissue elasticity component integrates the tissue/structure elasticity for the $0-8 \mathrm{~mm}$ layer behind the vaginal walls, from the depth comparative with the vaginal wall deformations in Test 1 and 3 (see Fig. 3 and parameter interpretation in Appendix 2 and [22]). The pelvic support component integrates the structure support from a depth of 5-45 mm, which is about the same as the vaginal wall deformations in Test 2 (see Fig. 3 and parameter interpretation in Appendix 2 and [27]).

Earlier, the intra- and inter-observer reproducibility for a set of ten vaginal tactile imaging markers was reported on 12 subjects [25]. Intra-observer intraclass correlation coefficients (ICC) were found in the range from 0.80 (Test 8: cough) to 0.92 (Test 3: rotation) with average value of 0.87 . Inter-observer ICCs were found in the range from 0.73 (Test 2, elevation pressure, and Test 8: cough) to 0.92 (Test 3: rotation) with average value of 0.82 . Intra-observer limits of agreement were in the range from $\pm 11.3 \%$ (Test 1) to $\pm 19.0 \% \%$ (Test 8 ) with average value of $\pm 15.1 \%$. 
Fig. 3 A diagram illustrating composition of BI-score from five components and VTI these components with specific weights parameters contributing to

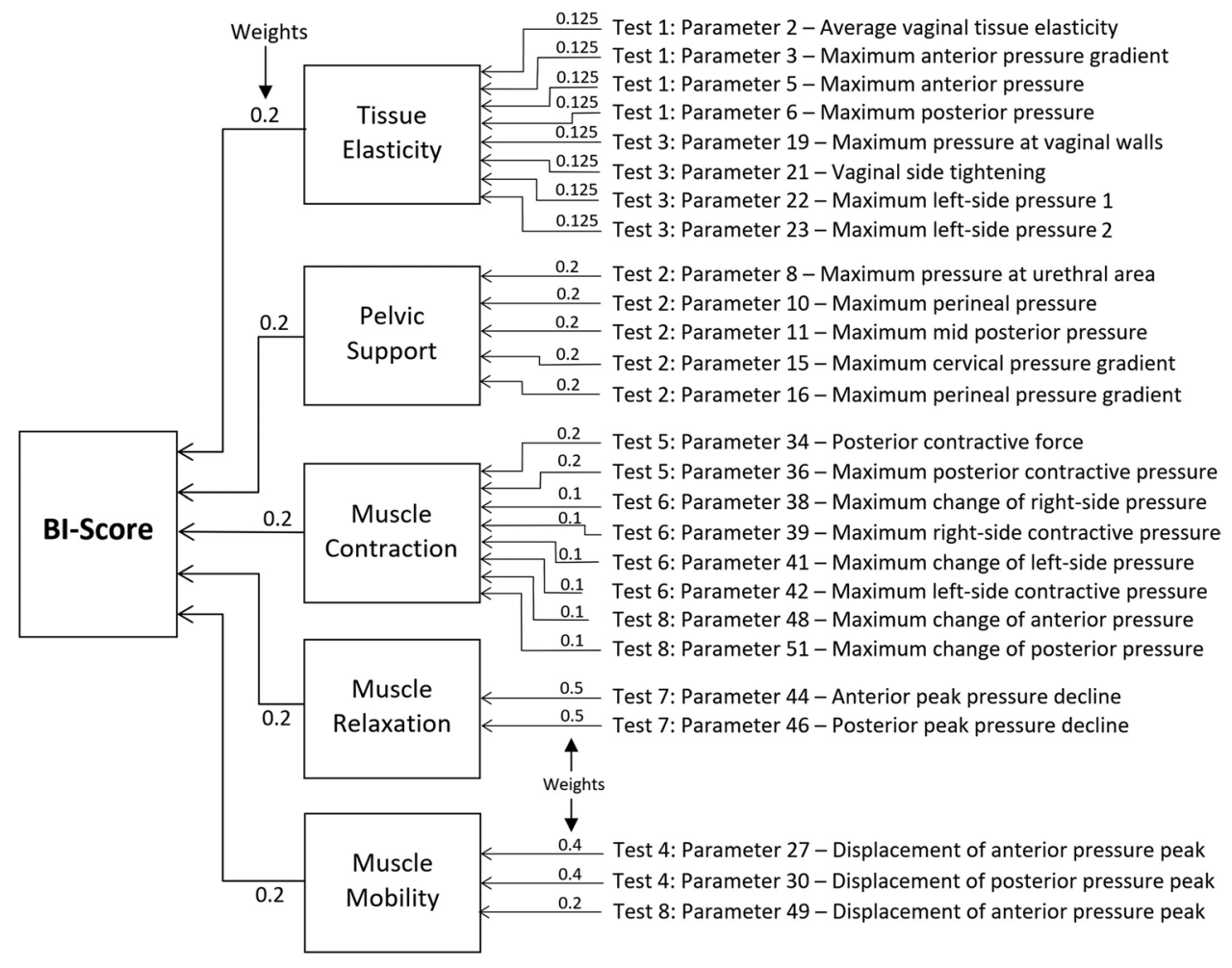

Fig. 4 BI-score calculated for normal (blue dots) and POP (red dots) cases against patient age for 253 cases analyzed in this study (left panel). BI-score boxplots for normal (blue dots) and POP (red dots) cases
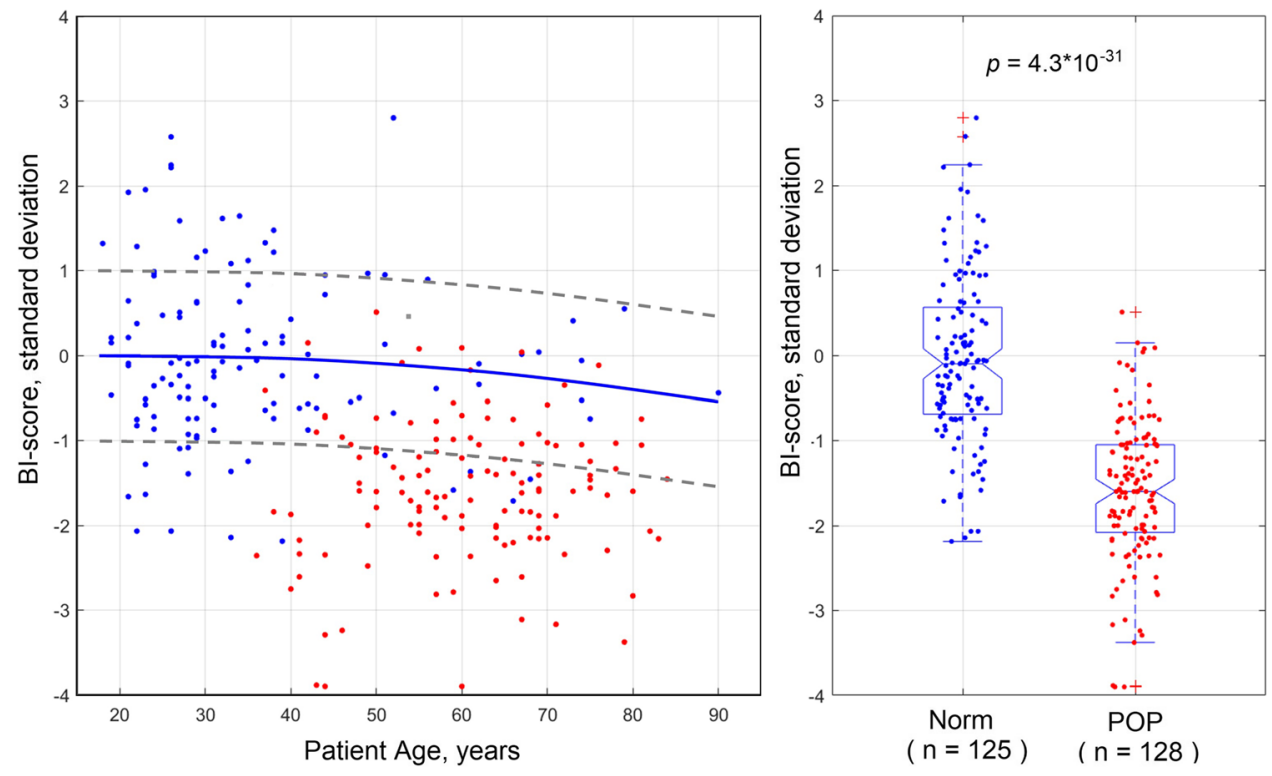

Inter-observer limits of agreement were in the range from $\pm 12.0 \%$ (Test 5 : voluntary contraction) to $\pm 26.7 \%$ (Test 2 : elevation) with average value of $\pm 18.4 \%$. These numbers lead to projection of reproductivity for the BI-score and its components in the range from \pm 0.1 to \pm 0.2 standard deviation. Improved inter-observer reproducibility is possible by additional operator training and consistency in VTI examination technique.
The tenth column in Table 2 shows VTI parameter changes in POP relative to the normal pelvic conditions. The elasticity parameters are decreasing by $-42.9 \% \ldots-55.1 \%$ in POP, the pelvic support parameters are decreasing by $-35.6 \% \ldots-46.0 \%$, and the muscle contraction parameters are decreasing by $-40.3 \% \ldots-55.7 \%$ in POP. The muscle relaxation speed parameters, which have a negative sign because muscle force involuntarily 
Fig. 5 An example of examination results with BI-score and its five components for a 58 -year-old patient with stage 2 anterior prolapse

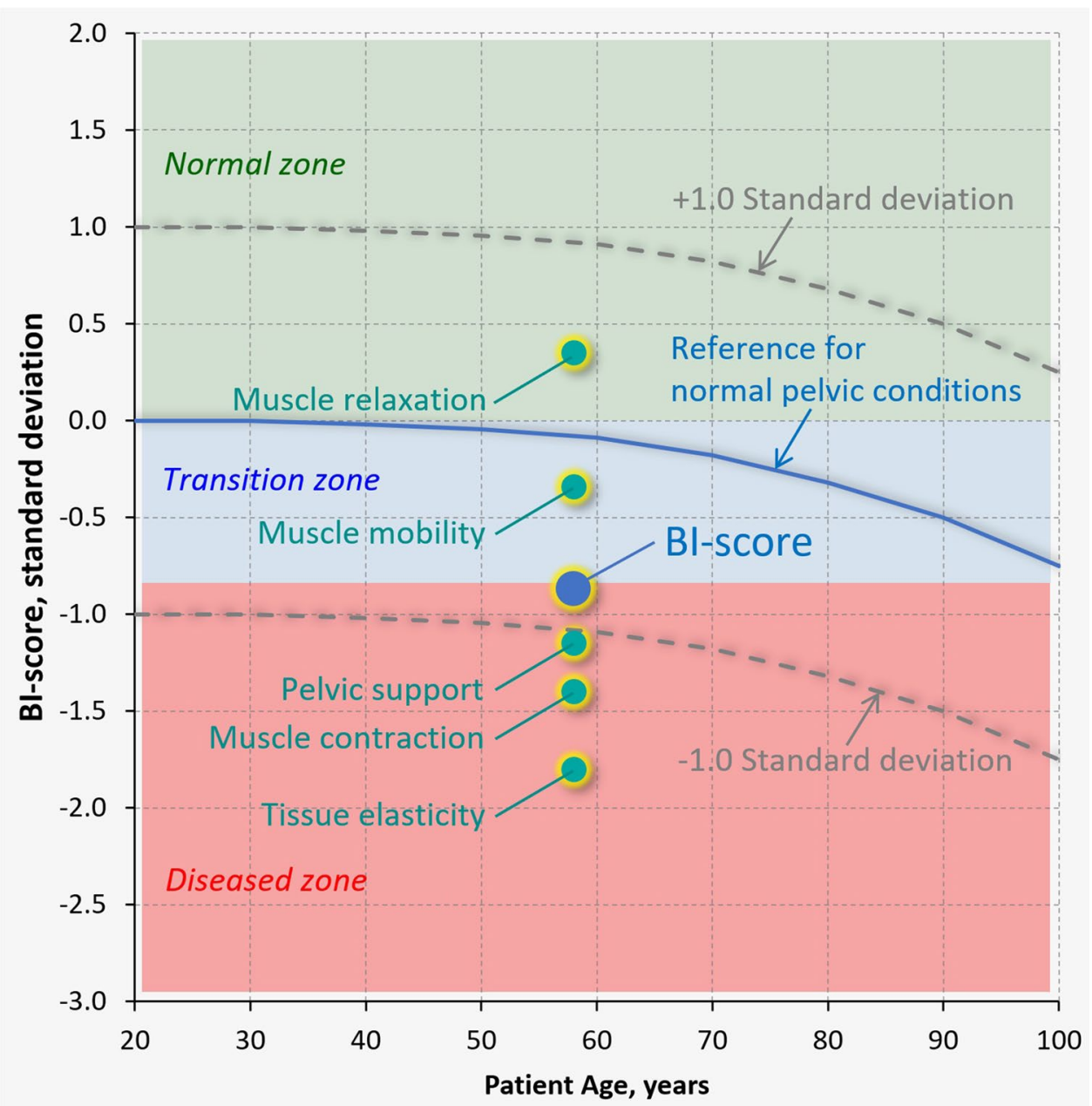

goes down, are increasing by $65.3 \%$ and $90.0 \%$ in POP_relaxation develops faster. The muscle mobility parameters, which may have a negative or positive sign, are increasing by $79.0 \%$ and $167.0 \%$ in POP_-muscle mobility develops along the vagina. All these results are expected and are in line with the previously reported results $[18,21,22]$.

The mean subject age and parity in the normal and POP groups are significantly different: 36 versus 65.5 years old and 0.9 versus 2.4 , respectively. That is the envisioned difference in the analyzed groups because for the reference (zero-line in the BI-score) we need a young population without POP which develops with age. Age-matched analysis for all normal versus all POP subjects aged from 38 to 52 years ( $p=0.48$ for age distributions) demonstrated $t$-test outcome for BI-score sensitivity to POP conditions with $p=2.1 \times 10^{-7}$. The mean subject weight and height are the same in both groups (see Table 1).

The last column in Table 2 brings $p$-values for the two-sample $t$-tests (normal versus POP). The $p$-values for the VTI parameters are found in the range of $1.2 \times 10^{-23}$ to $4.8 \times 10^{-2}$, most of the $p$-values being $<1.0 \times 10^{-5}$. The $p$-value for the BI-score has $p=4.3 \times 10^{-31}$ for two analyzed groups. It indicates that the data in these groups come from populations with unequal means and strong sensitivity to POP conditions. These results can be considered as statistically significant validation for the BI-score sensitivity to POP conditions. Since POP is often associated with concomitant pelvic floor disorders, including urinary and fecal incontinence, pelvic pain, voiding, and sexual dysfunctions [1, 29], and these disorders are thought to share common pathogeneses, tissue elasticity changes, weakening of the connective support tissues, and pelvic floor muscle dysfunction [9-14], the proposed BIscore may be used for the characterization of any of the above listed pelvic disorders and/or their combination.

Three colored backgrounds have been suggested to be used in the presentation of the patient examination results as shown in Fig. 5. The transition from green to blue background at BI-score $=0$ has sensitivity $=95.3 \%$ and specificity $=51.2 \%$ for the detection of POP conditions. The transition from blue to red background at BI-score $=-0.80$ has almost equalized 
sensitivity $=82.8 \%$ and specificity $=84.0 \%$ for diagnosing POP conditions. These transitions (BI-score $=0$ and BI-score $=-0.80$ ) are present in Fig. 5. The POP diagnostic accuracy of the BI-score, calculated as an area under a receiver operating characteristics (ROC) curve [30] for the analyzed sample, was found as $89.7 \%$. The dependence of the BI-score on age for normal pelvic conditions is described as a second order curve (see blue line in Fig. 5); its \pm standard deviations are depicted by the dashed curves. It is clear that an age-adjusted BI-score can also be calculated relatively to the normal blue curve in Fig. 5 similar to the Z-score in the bone densitometry.

As with bone density measurement, it is important to monitor patient progress with or without treatment. For this reason, it would be important to define the minimal clinically important difference (MCID) in BI-score. The future research directions also may address (1) BI-score use for monitoring of a pelvic floor treatment outcome, (2) obtaining periodic BI-scores before a woman has symptoms, (3) recommendation for specific treatment based on the five components (e.g., treatment for elasticity is needed but not for relaxation or muscle mobility), (4) predictive capabilities of the BI-score for symptoms (e.g., a woman is less or more likely to develop some form of pelvic floor dysfunction). These important questions are beyond the scope of this article.

The strength of this study is that the suggested BIscore covers biomechanical aspects of the pelvic floor, which include tissue elasticity, pelvic support, muscle contraction, involuntary relaxation, and mobility. All these aspects usually deteriorate as the pelvic disease develops. This quantitative characterization can be used in diagnosing and monitoring the pelvic conditions as well as in selecting and justifying a treatment.

The weakness of this study is the absence of statistically significant results for possible variations with ethnicity and race, which must be the subject of future research. Also, thousands of new VTI examinations for normal pelvic floor conditions may adjust the values of mean and standard deviation used in Eq. 1 for BI-score calculations.

\section{Conclusion}

The original purpose of this research was to systemize and simplify the presentation of the VTI examination results. Based on the analysis, it can be concluded that there are five components important for the biomechanical characterization of the pelvic floor. All these components contribute to the integral parameter the BIscore. Objectively measurable transformations of the pelvic tissues, support structures, and functions under different diseased conditions may be studied with the BI-score in future research and practical applications.

\section{Appendix 1.}

Table 3

Table 3 VTI examination procedure tests

\begin{tabular}{|c|c|c|}
\hline Test no. & Procedure & Output \\
\hline Test 1 & Probe insertion & $\begin{array}{l}\text { Tactile image* for vaginal anterior and posterior compartments along the entire vagina (resistance, } \\
\text { force, work, pressure gradients, tissue elasticity); } 3-15-\mathrm{mm} \text { tissue deformation }\end{array}$ \\
\hline Test 2 & Probe elevation & $\begin{array}{l}\text { Tactile image* for anterior and posterior compartments, which related to pelvic floor support } \\
\text { structures (pressure value and pressure gradients for specified/critical locations); 20-45-mm } \\
\text { tissue deformation }\end{array}$ \\
\hline Test 3 & Probe rotation & $\begin{array}{l}\text { Tactile images* for left and right sides along the entire vagina (force and pressure values for speci- } \\
\text { fied locations); 5-7-mm tissue deformation }\end{array}$ \\
\hline Test 4 & Valsalva maneuver & $\begin{array}{l}\text { Dynamic pressure response from opposite compartments (anterior vs. posterior) along the entire } \\
\text { vagina (changes in force and pressure; pressure peak displacements). }\end{array}$ \\
\hline Test 5 & Voluntary muscle contraction & $\begin{array}{l}\text { Dynamic pressure response from opposite compartments (anterior vs. posterior) along the entire } \\
\text { vagina (changes in force and pressure; maximum pressure values) }\end{array}$ \\
\hline Test 6 & Voluntary muscle contraction & $\begin{array}{l}\text { Dynamic pressure response from opposite sides (left vs. right) along the entire vagina (changes in } \\
\text { force and pressure; maximum pressure values) }\end{array}$ \\
\hline Test 7 & Involuntary relaxation & $\begin{array}{l}\text { Dynamic pressure response from opposite compartments (anterior vs. posterior) along the entire } \\
\text { vagina (changes in pressure) }\end{array}$ \\
\hline Test 8 & Reflex muscle contraction (cough) & $\begin{array}{l}\text { Dynamic pressure response from opposite compartments (anterior vs. posterior) along the entire } \\
\text { vagina (changes in force and pressure; pressure peak displacements). }\end{array}$ \\
\hline
\end{tabular}

*Probe maneuvers in Tests 1-3 are used to accumulate multiple pressure patterns from the tissue surface and create an integrated tactile image for the investigated area employing the image composition algorithms described in $[23,24]$ 


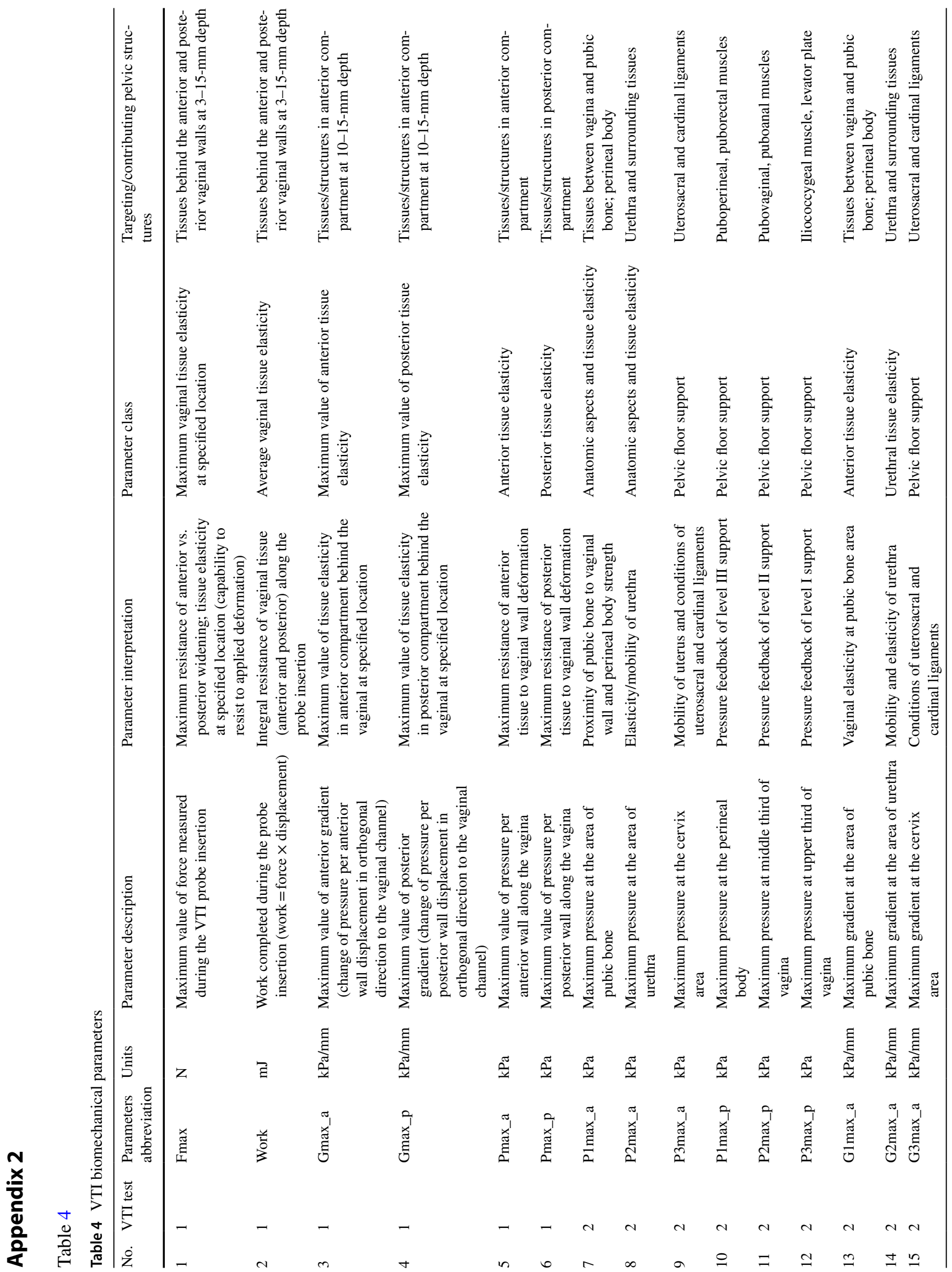




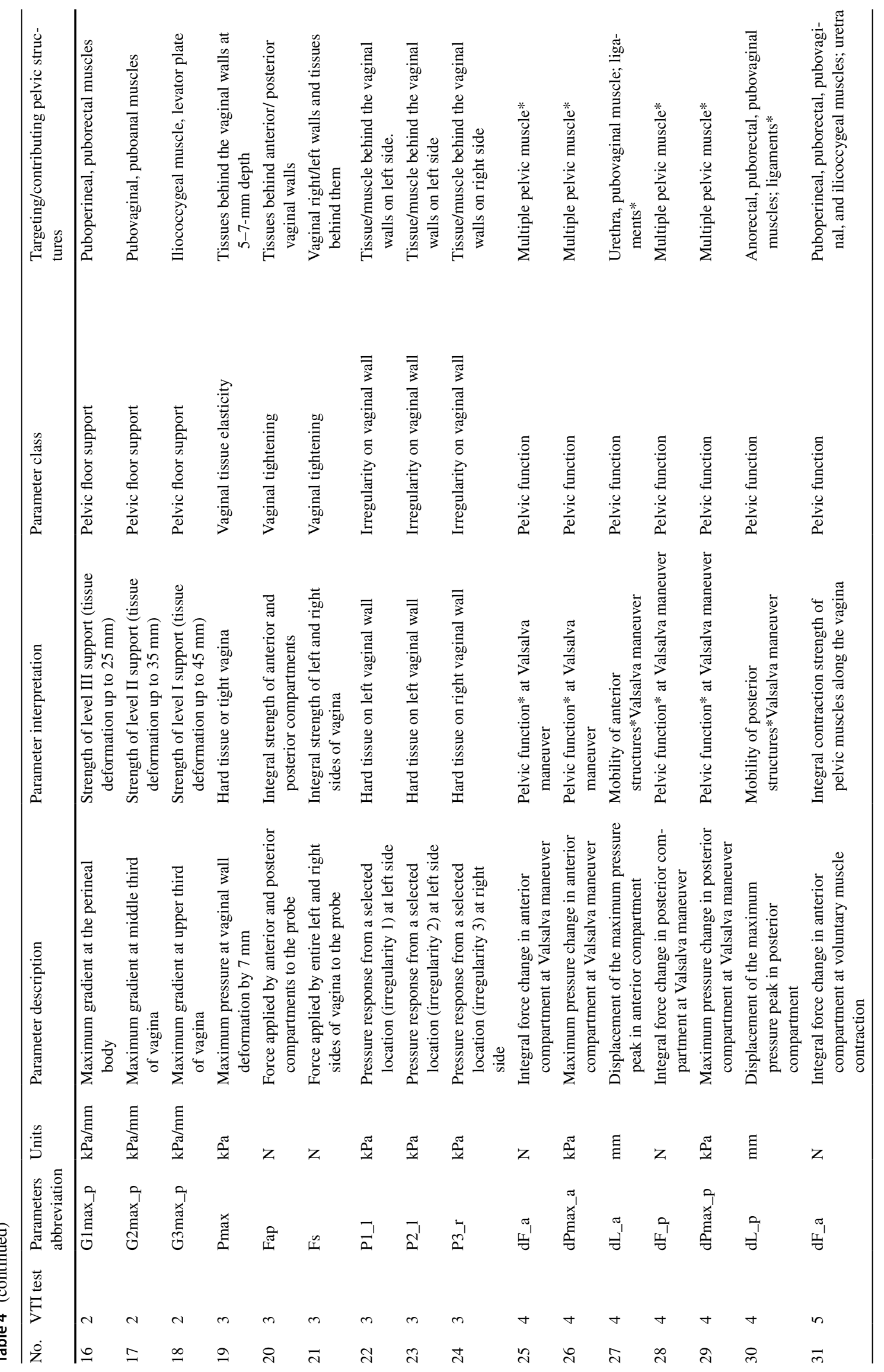




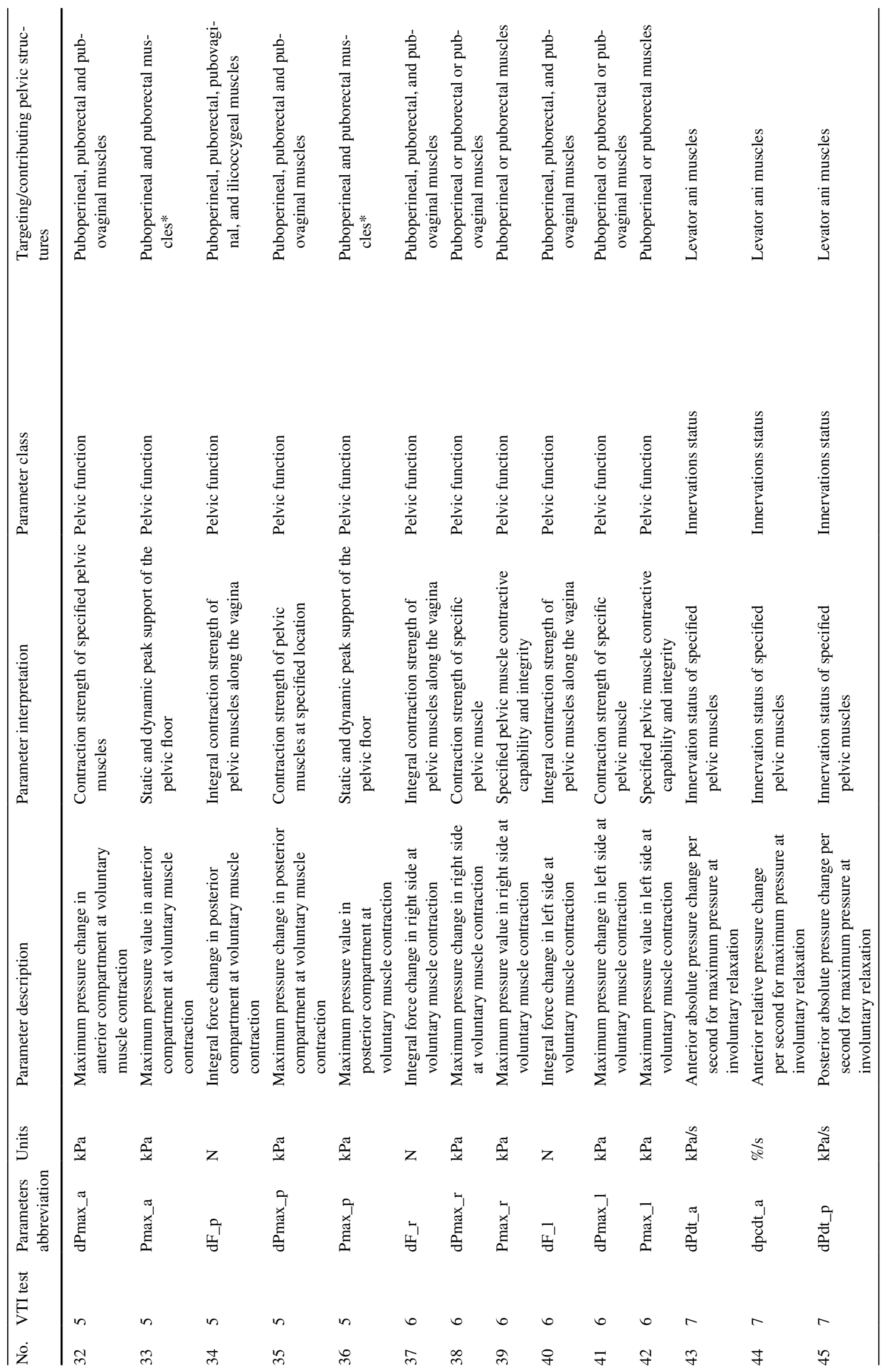




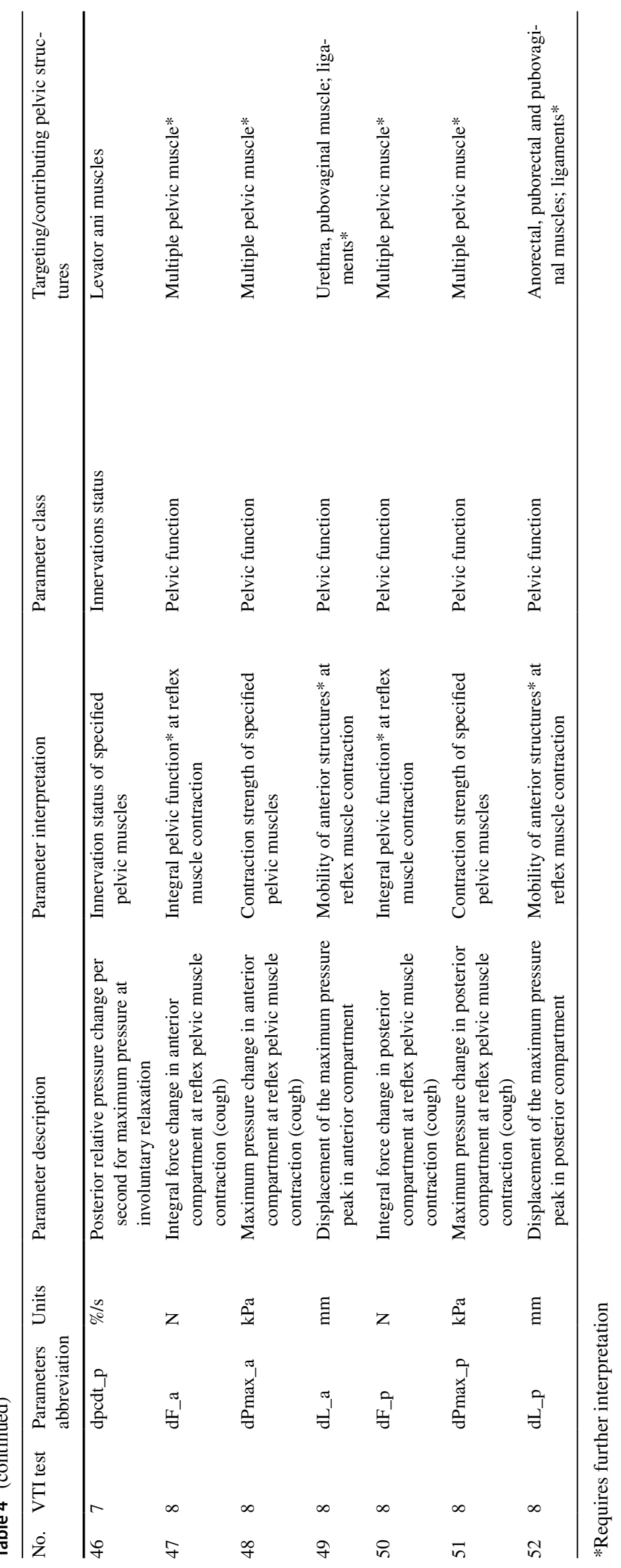


Acknowledgements The US Army Medical Research Acquisition Activity, 839 Chandler Street, Fort Detrick MD 21702-5014, is the awarding and administering acquisition office. Research reported in this publication was supported by the National Institute on Aging of the National Institutes of Health under award nos. R44AG034714 and SB1AG034714 and by the Department of Defense (DoD), through the Broad Agency Announcement (BAA), for Extramural Medical Research, under award no. W81XWH1920018. Opinions, interpretations, conclusions, and recommendations are those of the author and are not necessarily endorsed by the Department of Defense and the National Institutes of Health.

Contributions to authorship V Egorov: Project development, Device design and assembling, Imaging software development, Data analysis, Manuscript writing.

$\mathrm{H}$ van Raalte: Clinical protocol development, Data collection and analysis, Manuscript editing and review.

P Takacs: Clinical protocol development, Data collection and analysis, Manuscript editing and revision.

SA Shobeiri: Clinical protocol development, Data collection and analysis, Manuscript editing and revision.

V Lucente: Data collection and analysis, Manuscript editing and revision.

L Hoyte: Data collection and analysis, Manuscript editing and revision.

\section{Declarations}

Conflicts of interest Egorov is a CEO and a minor shareholder in Advanced Tactile Imaging, Inc. Egorov has submitted a patent application related to the reported approach. Raalte is a minor shareholder of Advanced Tactile Imaging, Inc. Takacs reports no conflict of interest. Shobeiri reports no conflict of interest. Lucente reports no conflict of interest. Hoyte reports no conflict of interest.

Open Access This article is licensed under a Creative Commons Attribution 4.0 International License, which permits use, sharing, adaptation, distribution and reproduction in any medium or format, as long as you give appropriate credit to the original author(s) and the source, provide a link to the Creative Commons licence, and indicate if changes were made. The images or other third party material in this article are included in the article's Creative Commons licence, unless indicated otherwise in a credit line to the material. If material is not included in the article's Creative Commons licence and your intended use is not permitted by statutory regulation or exceeds the permitted use, you will need to obtain permission directly from the copyright holder. To view a copy of this licence, visit http://creativecommons.org/licenses/by/4.0/.

\section{References}

1. The American College of Obstetricians (ACOG) and Gynecologists and the American Urogynecologic Society (AUGS). Pelvic organ prolapse. Practice Bulletin Number 185. Female Pelvic Med Reconstruct Surg. 2017;23(6):353-64.

2. Bump RC, Mattiasson A, Bo K, et al. The standardization of terminology of female pelvic organ prolapse and pelvic floor dysfunction. Am J Obstet Gynecol. 1996;175:10-7.

3. de Arruda GT, Dos Santos HT, Virtuoso JF. Pelvic floor distress inventory (PFDI)-systematic review of measurement properties. Int Urogynecol J. 2021;32(10):2657-69.

4. Okobi OE. A systemic review on the association between infertility and sexual dysfunction among women utilizing female sexual function index as a measuring tool. Cureus. 2021;13(6):e16006.
5. The American College of Obstetricians (ACOG) and gynecologists and the American Urogynecologic Society (AUGS). Pelvic organ prolapse. Interim update. Female Pelvic Med Reconstruct Surg. 2019;25(6):397-408.

6. The American College of Obstetricians (ACOG) and gynecologists and the American Urogynecologic Society (AUGS). Urinary incontinence in women. Practice bulletin number 155. Obstet Gynecol. 2015;126(5):e66-81.

7. Silva AC, Maglinte DD. Pelvic floor disorders: what's the best test? Abdom Imaging. 2013;38(6):1391-408.

8. Swamy N, Bajaj G, Olliphant SS, Henry JA, Jambhekar K, Pandey T, Ram R. Pelvic floor imaging with MR defecography: correlation with gynecologic pelvic organ prolapse quantification. Abdom Radiol (NY). 2021;46(4):1381-9.

9. Quaghebeur J, Petros P, Wyndaele JJ, De Wachter S. Pelvic-floor function, dysfunction, and treatment. Eur J Obstet Gynecol Reprod Biol. 2021;26(5):143-9.

10. Kieserman-Shmokler C, Swenson CW, Chen L, Desmond LM, AshtonMiller JA, DeLancey JO. From molecular to macro: the key role of the apical ligaments in uterovaginal support. Am J Obstet Gynecol. 2020;222(5):427-36.

11. Johnston SL. Pelvic floor dysfunction in midlife women. Review Climacteric. 2019;22(3):270-6.

12. Lukacz ES, Santiago-Lastra Y, Albo ME, Brubaker L. Urinary incontinence in women: a review. JAMA. 2017;318(16):1592-604.

13. Gong R, Xia Z. Collagen changes in pelvic support tissues in women with pelvic organ prolapse. Eur J Obstet Gynecol Reprod Biol. 2019;234:185-9.

14. Cardenas-Trowers O, Meyer I, Markland AD, Richter HE, Addis I. A review of phytoestrogens and their association with pelvic floor conditions. Female Pelvic Med Reconstr Surg. 2018;24(3):193-202.

15. Gynecologic Health and Disease Branch at Eunice Kennedy Shriver National Institute of Child Health \& Human Development (NICHD, $\mathrm{NIH}$ ), Branch Research Priorities. Non-invasive diagnostic and screening tools. Accessed October 20, 2021. https://www.nichd.nih.gov/about/org/ der/branches/ghdb

16. Easley DC, Abramowitch SD, Moalli PA. Female pelvic floor biomechanics: bridging the gap. Curr Opin Urol. 2017;27(3):262-7.

17. Siddiqui NY, Gregory WT, Handa VL, et al. American Urogynecologic Society prolapse consensus conference summary report. Female Pelvic Med Reconstr Surg. 2018;24(4):260-3.

18. Lucente V, van Raalte H, Murphy M, Egorov V. Biomechanical paradigm and interpretation of female pelvic floor conditions before a treatment. Int J Women's Health. 2017;9:521-50.

19. Hoyte L, Damaser MS. What the future holds, in: biomechanics of the female pelvic floor. Elsevier. 2016:435-6.

20. Ahmad AN, Hainsworth A, Williams AB, Schizas AM. A review of functional pelvic floor imaging modalities and their effectiveness. Clin Imaging. 2015;39(4):559-65.

21. Egorov V, van Raalte H, Lucente V, Sarvazyan A. Biomechanical characterization of the pelvic floor using tactile imaging. In: Biomechanics of the Female Pelvic Floor, Eds. Hoyte L, Damaser MS, Elsevier. 2016: 317-48.

22. Egorov V, Shobeiri AS, Takacs P, Hoyte L, Lucente V, van Raalte H. Biomechanical mapping of the female pelvic floor: prolapse versus normal conditions. Open J Obstetrics and Gynecol. 2018;8:900-25.

23. Egorov V, Ayrapetyan S, Sarvazyan AP. Prostate mechanical imaging: 3-D image composition and feature calculations. IEEE Trans Med Imaging. 2006;25(10):1329-40.

24. Egorov V, Sarvazyan AP. Mechanical imaging of the breast. IEEE Trans Med Imaging. 2008;27(9):1275-87.

25. van Raalte H, Lucente V, Ephrain S, Murphy M, Bhatia N, Sarvazyan N, Egorov V. (2016) intra- and inter-observer reproducibility of vaginal tactile imaging. Female Pelvic Med Reconstr Surg. 2016;22:S130-1. 
26. McGill R, Tukey JW, Larsen WA. Variations of box plots. Am Statistician. 1978;32:12-6.

27. Faulkner KG. The tale of the T-score: review and perspective. Osteoporos Int. 2005;16(4):347-52.

28. Kanis JA. Assessment of fracture risk and its application to screening for postmenopausal osteoporosis: synopsis of a WHO report. WHO Study Group. Osteoporos Int. 1994;4(6):368-81.

29. Patterson D, Handa VL. Pathophysiology of pelvic floor disorders. In: Urogynecology and reconstructive pelvic surgery. Philadelphia: Elsevier; 2022. p. 70-81.
30. Hajian-Tilaki K. Receiver operating characteristic (ROC) curve analysis for medical diagnostic test evaluation. Caspian J Intern Med. 2013;4(2):627-35.

Publisher's note Springer Nature remains neutral with regard to jurisdictional claims in published maps and institutional affiliations. 